\title{
Characterization of an IR-UWB Transceiver Under Radiation Conditions
}

\author{
Andre Lübken, Martin Drobczyk, Jan Budroweit \\ Department of Avionics Systems \\ Institute of Space Systems, German Aerospace Center (DLR) \\ 28359 Bremen, Germany \\ \{andre.luebken, martin.drobczyk, jan.budroweit\}@dlr.de
}

\begin{abstract}
In this paper, the characterization of an impulse radio ultra-wideband (IR-UWB) radio frequency transceiver in a radiation environment is presented. IR-UWB is a well-known terrestrial technology for wireless communication and localization with interesting features also for space applications. The currently available hardware is Commercial-of-the-Shelf and it is essential to analyze the performance under radiation conditions to utilize the transceiver in reliable space applications, specifically intra-spacecraft communication. The device under test is exposed to $\gamma$-rays to evaluate total ionizing dose effects. Another test campaign is performed to analyze the single event effects induced by protons. The test procedure and results are presented and show that the transceiver withstands typical low earth orbit radiation conditions without significant degradation or malfunction.
\end{abstract}

Keywords-IR-UWB, Intra-spacecraft communication, TID, SEE, Radiation environment, ,

\section{INTRODUCTION}

For several years now, wireless technologies have been getting increased attention in the space community by providing wireless communication networks in the spacecraft and launcher segment [1]. Also, the German Aerospace Center (DLR) has been focusing on the establishment of wireless technologies in space applications, in particular intra-vehicle communications. Several candidates were analyzed and the IR-UWB was identified as a technology with huge potential since it fulfills the very stringent electromagnetic requirements when operating in metallic enclosures with other radio frequency (RF) sensitive systems. Also, it is resilient to multipath fading, which is an ideal characteristic in highly reflective environments. DLR has been implementing several projects, where it demonstrated the successful utilization of IR-UWB. One of these projects was the deployment of an IRUWB network on the International Space Station, called Wireless Compose [2]. DLR also works on the development of higher-layer protocols to be operated with the IR-UWB standard to allow a low-latency and deterministic communication between spacecraft components [3]. However, the establishment can only succeed when the technology is sufficiently reliable when operated in a space environment. Especially, radiation is a challenging factor when using Commercial-of-the-Shelf (COTS) components in space. COTS components are often assumed to experience problems when the dose exceeds $15 \operatorname{krad}(\mathrm{Si})$, whereas specially manufactured radiation hardened components have tolerances in the range of 50-300 $\mathrm{krad}(\mathrm{Si})$. On the other hand, COTS components are cheap and easily available.

Also, the currently available IR-UWB RF devices are COTS hardware, and thus, it is important to characterize them in a radiation environment. The results are essential to analyze the future utilization of the tested hardware in a reliable environment as space applications mostly require.

In this paper, we characterize the DW1000 UWB transceiver to evaluate the performance concerning the total ionizing dose (TID) and single event effects (SEE). The TID is accumulated during the mission lifetime in space. A typical TID of max. $1.87 \mathrm{krad}(\mathrm{Si})$ can be expected per year in an exemplary heliosynchronous low earth orbit (LEO) at an altitude of $800 \mathrm{~km}$ [4]. It has an irreversible destructive effect on the hardware which leads to total damage of the hardware electronics due to the induced charge in the electronic layers. Moreover, SEEs can lead to bit flips and latch-ups, which are reversible but also SEEs can damage the hardware electronics.

\section{DEVICE UNDER TEST}

The selected device under test (DUT) is the DW1000 [5] IR-UWB transceiver by Decawave.

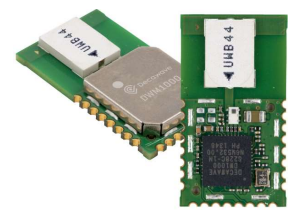

Fig. 1. Picture of a Decawave DWM1000 DUT

The DW1000 implements the ultra-wideband physical layer (PHY) of the IEEE 802.15.4 [6] standard for wireless sensor networks and it operates at frequencies from 3.5 to 6.5 
GHz. The chip is a comparatively cheap commercial-of-theshelf (COTS) component, designed for time-of-flight localization applications with accuracy in the centimeter range and mostly marketed for industrial asset tracking use cases.

\section{TEST REQUIREMENTS AND CONDITIONS}

\section{A. Test requirements}

Two test campaigns have been carried out on the DW1000 transceiver. For the first test purpose (2016), the primary goal was to achieve a total ionizing dose (TID) of $>20 \mathrm{krad}(\mathrm{Si})$ to fulfill the test level requirement of the ESCC-22900 [7]. Two samples were irradiated and tested in an in-situ configuration. The required sample number (10) according to the ESCC22900 was reduced due to the limited test equipment, test time and laboratory space. The standard dose rate window required by the ESCC is between 0.36 and $180 \mathrm{krad}(\mathrm{Si}) / \mathrm{h}$. Low dose rate tests were not intended. For the second test (2018), three samples have been irradiated to a total dose of $180 \mathrm{krad}(\mathrm{Si})$ each.

To evaluate the response of single event effects (SEE), the DUT has been irradiated with protons of different energies (70 MeV to $184 \mathrm{MeV}$ ). Similar to the TID test, two samples have been investigated but due to time constraints and unexpected behaviors under different energies.

For both tests, the test was performed in air at ambient temperature $\left(20 \pm 10^{\circ} \mathrm{C}\right)$.

\section{B. Test sites}

The selected test facility for TID purposes is the cobalt- 60 source of Helmholtz-Zentrum Berlin (HZB) in Potsdam, Germany [8]. Cobalt-60 decays into nickel- 60 by emitting $\beta$ and $\gamma$-rays. The emitted $\gamma$-rays are used for irradiation purposes. A picture of the test facility is presented in Figure 2 , with the radiation source illustrated in the red-dotted frame. The source can be pulled-up to enable radiation. The dose rate or fluence depends on the distance to the source. The achieved TID is determined by the dose rate and irradiation time.

The SEE proton characterization has been carried out at the Kernfysisch Versneller Instituut (KVI) in the Netherlands using the accelerator providing a primary beam energy of 184 $\mathrm{MeV}$ at the DUT surface. Other energies are provided using a set of aluminum degraders. The adapted energies used for this test are 150, 120 and $70 \mathrm{MeV}$.

\section{Test setup}

\section{1) $T I D$}

The DUT samples are located at a distance to the source to achieve a dose rate of $1 \mathrm{krad} / \mathrm{h}$ in Silicon on the first test campaign. For the second test campaign, a closer distance was selected with an appropriated dose rate of approx. 11.8 $\operatorname{krad}(\mathrm{Si}) / \mathrm{h}$.

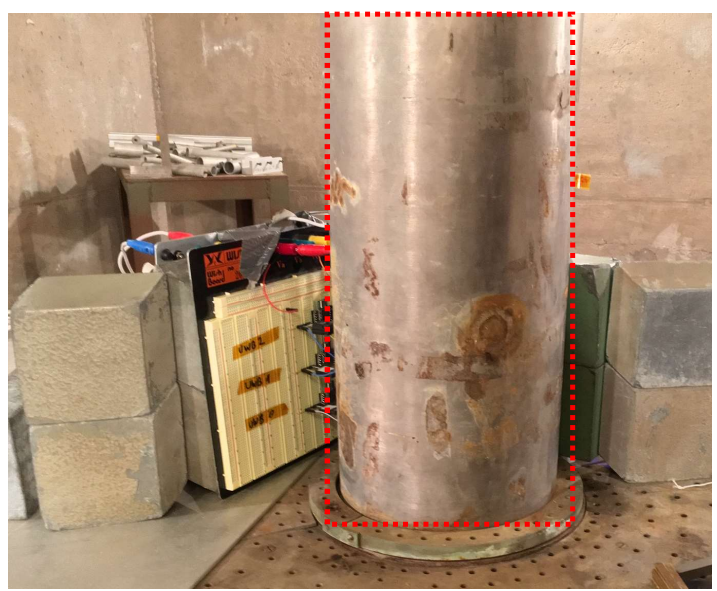

Fig. 2. Picture of the cobalt- 60 source and sample setup of the HZB. Red-dotted frame shows the cylinder the source can be pulled up into to enable irradiation.

The samples are powered individually by an external power supply and the voltage and a data acquisition unit placed in the control area outside the irradiation room that monitors current conditions. For the first test in 2016, the samples were operated in idle-mode without data analysis on the packet transmission and reception. One sample consisted of the single DWM1000 RF module, which integrates the DW1000 IC and its needed peripherals, and another sample contains the RF module attached to the STM32L151 ARM Cortex M3 Microcontroller. The primary objective was to evaluate the supplied current with an increased TID. Due to the predefined test duration, the test was limited to a max. radiation dose of $27.5 \mathrm{krad}(\mathrm{Si})$. For this reason and due to the strong performance, a second test in 2018 was performed with an extended dose level to search for any damage thresholds. Here, three single DWM1000 samples were operated in a nominal mode where data is being transmitted from all three samples to a shielded receiver unit. The corresponding microprocessors to operate the samples under test were shielded with lead bricks to minimize radiation effects on this part.

2) $S E E$

For the SEE test, the setup consists of three DUTs mounted on a motorized frame to be moved into the proton beamline one by one. The DUT is soldered on a commercially available evaluation board including an STM32L151 ARM Cortex M3 Microcontroller (Radino32_DW1000) [9]. The controller holds the necessary firmware to run the test procedure and collect diagnostic data from the DUT during the test. Two additional receiver nodes were placed in the test chamber at a distance of $4.75 \mathrm{~m}$ each from the DUT. The DUT acted as an initiator for network diagnostic tests as well as for time of flight ranging measurements. Both DUTs for this test have the lot code NOVC55.00 and a manufacturing date in Oct. 2017.

All nodes are connected to programmable power supply units (PSU) and are providing their diagnostic data via serial data connections to a PC running custom experiment control 
software. The experiment controller ensures that only the DUT moved into the beamline is powered and triggers hard resets via the power cycling of the PSUs in case a node does not provide its diagnostic data in the expected format or no data at all.

This system is accessible from the safety of the control room and allows the operator to configure the nodes for the next experiment. This also alleviates the need to access the test chamber in between test runs as higher dose rates caused by the activation of objects in the chamber mandate a cooldown period before safe access is possible again. A diagram of the test setup is shown in Fig. 3.

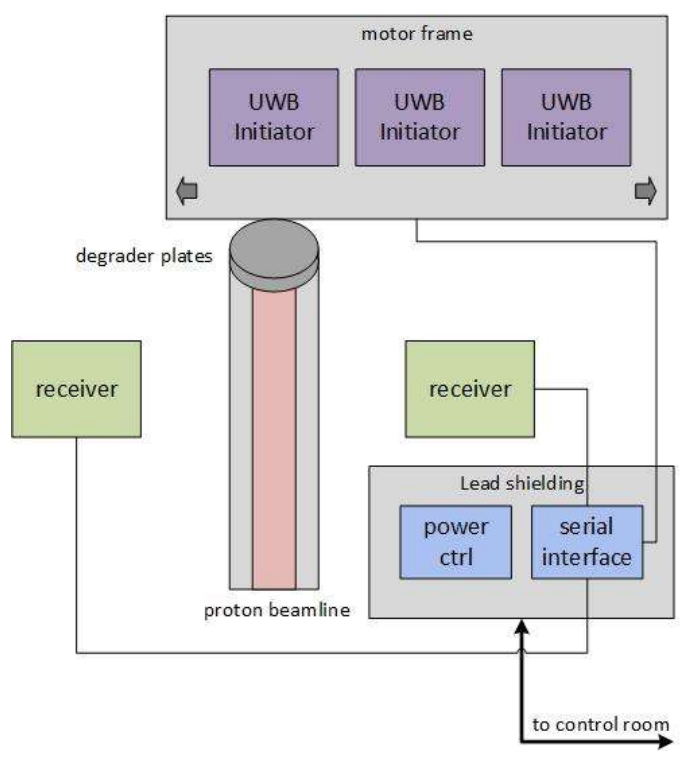

Fig. 3. Test setup for the SEE tests.

The UWB transceivers are configured to use a $110 \mathrm{kbit} / \mathrm{s}$ physical data rate as per the IEEE 802.15.4 [6] standard. The low data rate is used to ensure a stable connection so that possible data loss can be attributed to the irradiation, e.g. because a receive buffer register flipped and corrupted the data.

Diagnostic data is produced after each frame transmission indicating successful transmission, errors or missing frames in addition to the ranging data. Register error events as well as various parameters on the RF performance of the IR-UWB link, voltage levels and the on-chip temperature sensor are logged as well. This process is repeated every $150 \mathrm{~ms}$ for the duration of the experiment.

\section{Test procedure and evaluation methods}

\section{1) $T I D$}

For the first TID test campaign, the DUT consisting of two separate samples was operated in idle-mode. One of the samples was operated by a microcontroller, which was also in the radiation beam.

In the second test campaign, the samples were operated by separate microcontroller boards and they transmitted data packets to a receiver unit. Both, the microcontroller boards as well as the receiver unit were shielded with lead bricks to minimize the radiation impact and to allow a more detailed effects analysis based on the packet reception rate during the radiation campaign. Each time the receiver unit received a packet of one of the three samples, it toggled a generalpurpose input/output (GPIO) signal to monitor the packet delivery, which was indicated by an increased voltage level, see Fig. 4. In case that the current conditions are running out of the device's specification or the target dose is achieved, the test is halted and possibly stopped.

2) $S E E$

The SEE test was performed at different energy and particle flux levels. Experiments were conducted at 184, 150, 120 and $70 \mathrm{MeV}$, with a mean flux level chosen to achieve a balance between the probability of error occurrence and usability of the resulting diagnostic data. Higher mean flux combined with high energy yields frequent system lockups that make hard resets of the system necessary. If these occur in rapid succession, the control system is not able to capture meaningful data.

After setup is complete all nodes are powered and are given a burn-in time before the experiment commences to
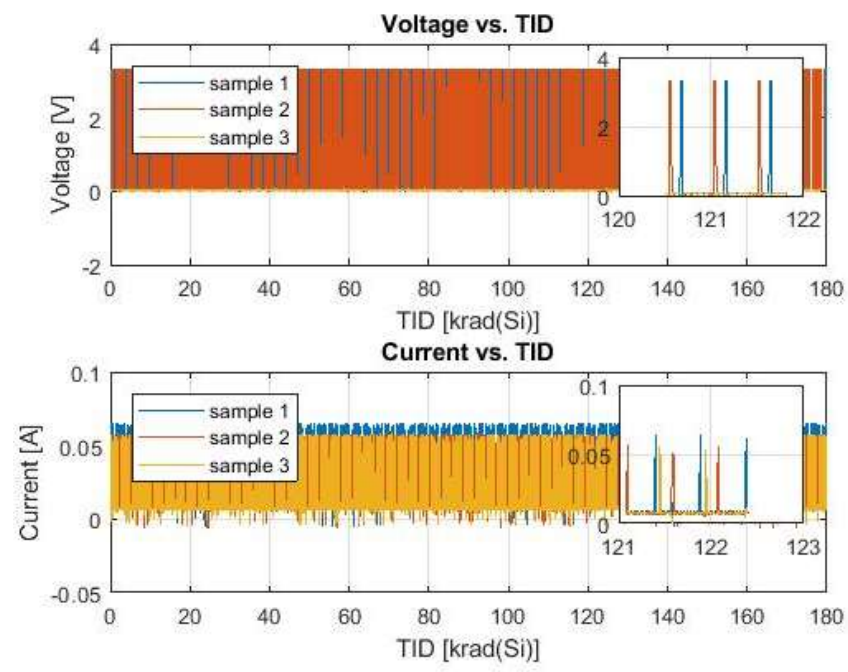

Fig. 4. Applied current levels (Tx) voltage levels (Rx) vs. TID on DUT sample 1 at the second test campaign in 2018

ensure that temperature effects due to heating especially of the receivers, which are most of the time in listen mode, do not affect the readout of the transceiver diagnostic data throughout the experiment.

The node (DUT) in this configuration is periodically transmitting a poll message to the two receiver nodes which in turn initiate the ranging procedure using a single-sidedtwo-way-ranging scheme. After completion of the range measurements, all nodes perform a check of the DW1000 transceiver ICs data registers and compare their values to a known good state. If errors are detected, a rewrite of the 
respective registers is attempted. If this check fails or if the register is part of certain registers that are deemed critical, the microcontroller will attempt a soft reset of the transceiver IC. If these attempts fail to correct the errors a hard reset is performed by power cycling the nodes PSU channel. However, the microcontrollers commanding the transceiver ICs, especially of the DUT which is moved into the beamline together with the DW1000, can lock up and will not produce any serial output for the experiment controller. In this case, the experiment controller software treats the periodic messages indicating the transmission state of the last data packet sent via the IR-UWB link as a heartbeat signal. If a report by one of the nodes is missing, the experiment controller will issue a hard reset of that node by power cycling the respective PSU channel.

The data logged by the experiment controller on the transceivers register errors indicate the exact register, expected and the actual content. For the results, this was accumulated into classes of critical errors, which indicate non-recoverable changes that mandate a reset of the transceiver, non-critical errors that can be resolved by rewriting the register and errors found in the one-time programmable memory (OTP) of the IC. RF diagnostic data yields estimated received power and first path power estimates since due to the pulsed nature of the signal it is not possible to measure a received signal strength indication (RSSI) using IR-UWB directly.

\section{TEST RESUlTS AND DisCUSSION}

In this section, selected results of the TID and SEE tests are presented.

\section{A. TID}

The primary goal of the first TID test is to verify the device behavior under $\gamma$-rays up to a total dose of $>20 \mathrm{krad}(\mathrm{Si})$ only on the supplied current in idle-mode.

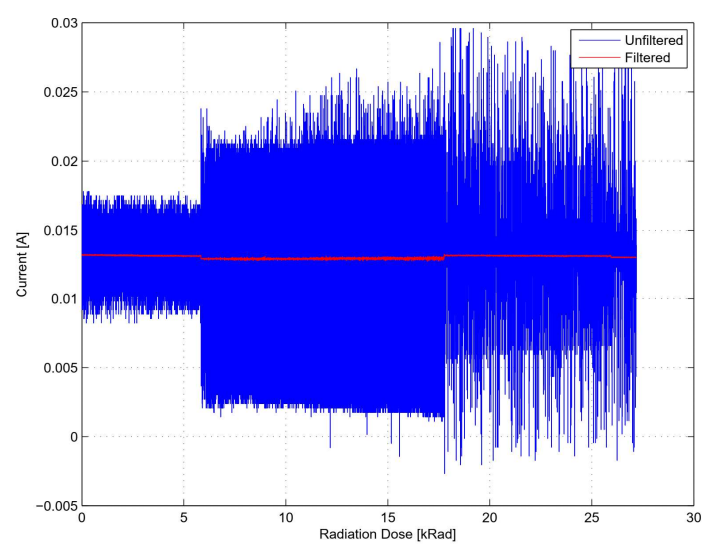

Fig. 5. Applied current conditions vs. TID on DUT sample 1

The current of the DUT (sample 1) exceeded this target level without any anomalies and the test was stopped at 27.5 $\operatorname{krad}(\mathrm{Si})$.
The test results are presented in Fig. 5. The fluctuations in the current levels indicate the different test intervals and the associated data resolutions. However, the filtered (averaged) value remained approximately at the same level during the complete test period. The current of sample 2 in Fig. 6 also includes the microcontroller, which was part of the DUT as well. In contrast to sample 1 , the current levels show that the microcontroller current abruptly drops at approx. 26.2 $\operatorname{krad}(\mathrm{Si})$, but the idle-mode current of the UWB module can be still measured. The observations were also validated with a hardware functional check after completion of the test campaign and they also showed that samples 1 and 2 were still operable without any measurable degradation and the used microcontroller was damaged by the achieved TID.

In the second test campaign, the samples were operated in transmit mode and the supporting microcontrollers, as well as the receiver units, were located behind a lead brick to minimize the radiation impact on the supporting hardware. The receiver unit recognized a $100 \%$ packet loss of sample 3 at approx. $88.2 \mathrm{krad}(\mathrm{Si})$.

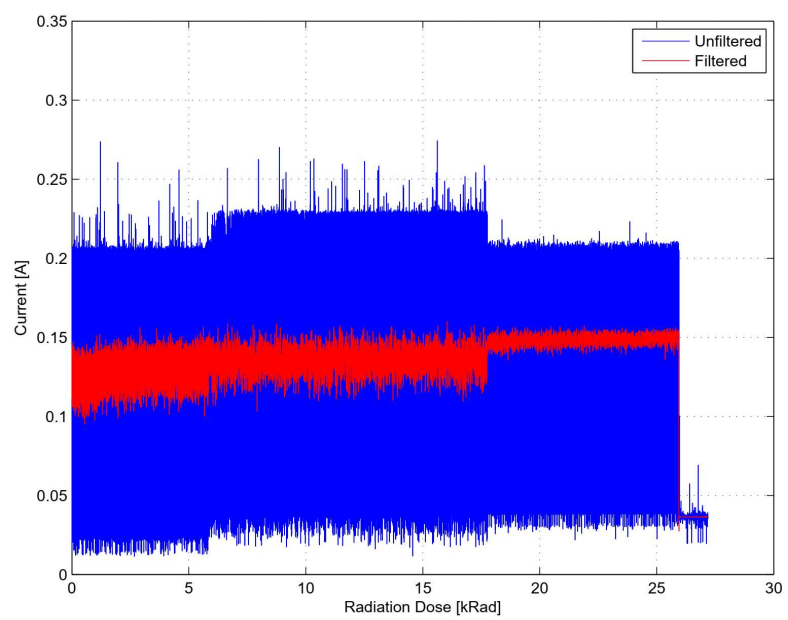

Fig. 6. Applied current conditions vs. TID on DUT sample 2

Moreover, the current level of sample 3 started to fluctuate and increase with the first significant peak of $0,1 \mathrm{~A}$ at 94 $\operatorname{krad}(\mathrm{Si})$. At $96.4 \mathrm{krad}(\mathrm{Si})$ the fluctuations dominate the current measurement and indicate a destructive effect on the sample, see Fig. 7 In contrast, samples 1 and 2 operated without any degradation effects on the packet delivery ratio (PDR) until the test was finished at $180 \mathrm{krad}(\mathrm{Si})$. However, this does not mean that no bit errors occurred, since the DW1000 RF IC works with Forward Error Correction (FEC) on the physical layer. A bit error increase due to radiation degradation is thus not detectable in this test setup because of the intact error correction. 


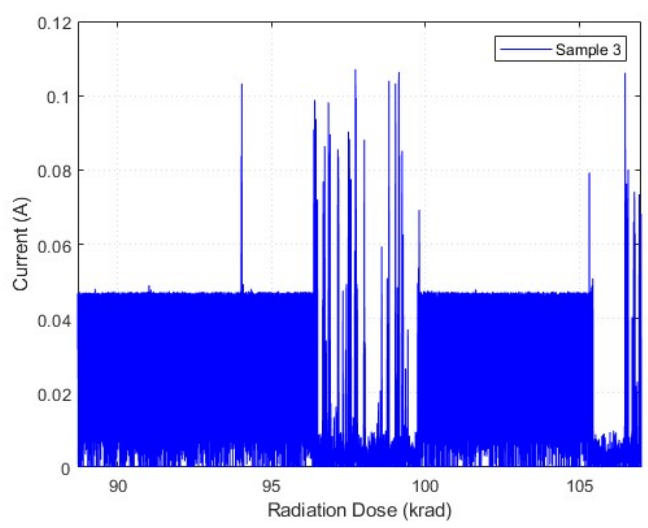

Fig. 7. Applied current conditions vs. TID on DUT sample 3

\section{B. SEE}

The single event effect results mostly show a high number of forced hard resets by the experiment controller, where the DUT is power cycled, either because it did not report to the experiment controller at all or the message format was incorrect. This is, in contrast, to register errors on the UWB IC itself and suggests that the controlling microcontroller is more susceptible to the irradiation effects.

Table I shows the energy, flux and fluence levels used for the different tests, while Table II summarizes the results. Deviating from ESCC 25100 a lower fluence was chosen in combination with a maximum possible flux that yielded a manageable error rate. Higher fluences led to frequent lock ups of the controlling microcontroller, making the read out of the transceiver diagnostic data impossible. Noticeable is the number of errors in the OTP memory of the transceiver. For this evaluation, multiple error reports of the same OTP register address within the same reset period have been filtered out, as these registers cannot be rewritten by the firmware without losing the ability to write to them.

TABLE I. TEST CONFIGURATION

\begin{tabular}{|c|c|c|c|c|}
\hline $\begin{array}{c}\text { Test } \\
\#\end{array}$ & DUT & $\begin{array}{c}\text { Energy } \\
(\text { MeV })\end{array}$ & $\begin{array}{c}\text { Fluence } \\
\left(1 / \text { cm }^{2}\right)\end{array}$ & $\begin{array}{c}\text { Mean Flux } \\
\left(1 / \mathbf{c m}^{2} / \mathbf{s}\right)\end{array}$ \\
\hline$\# 1$ & $\# 1$ & 184 & $1.05 \times 10^{9}$ & $4.07 \times 10^{5}$ \\
\hline$\# 2$ & $\# 1$ & 150 & $1.0 \times 10^{10}$ & $3.58 \times 10^{6}$ \\
\hline$\# 3$ & $\# 1$ & 120 & $1.0 \times 10^{10}$ & $1 \times 10^{7}$ \\
\hline$\# 4$ & $\# 2$ & 70 & $4.8 \times 10^{10}$ & $4.16 \times 10^{7}$ \\
\hline$\# 5$ & $\# 2$ & 70 & $1.0 \times 10^{10}$ & $6.38 \times 10^{6}$ \\
\hline
\end{tabular}

Nevertheless, they comprise a significant amount of the total errors detected when considering that they only represent a small area of the die compared to the regular registers which could be explained by the different cell design employed to construct them. Interestingly, although the content readout of the register was changed by the irradiation, the effuse were not blown after a hard reset.
TABLE II. DUT REGISTER ERRORS

\begin{tabular}{|c|c|c|c|c|}
\hline \multirow{2}{*}{$\begin{array}{c}\text { Test } \\
\#\end{array}$} & \multirow{2}{*}{$\begin{array}{c}\sigma \\
\text { Hard resets } \\
\left(\mathrm{cm}^{2} / \text { dev }\right)\end{array}$} & \multicolumn{3}{|c|}{$\sigma$ Errors } \\
\hline & & OTP & Critical & Non-critical \\
\hline$\# 1$ & $3.6 \times 10^{-8}$ & $1.6 \times 10^{-8}$ & $1.9 \times 10^{-9}$ & 0 \\
\hline$\# 2$ & $5.2 \times 10^{-8}$ & $5.3 \times 10^{-9}$ & $1.0 \times 10^{-9}$ & $1.0 \times 10^{-10}$ \\
\hline$\# 3$ & $2.6 \times 10^{-8}$ & $1.6 \times 10^{-9}$ & $1.3 \times 10^{-9}$ & $3.0 \times 10^{-10}$ \\
\hline$\# 4$ & $7.3 \times 10^{-9}$ & 0 & $3.54 \times 10^{-10}$ & $6.25 \times 10^{-11}$ \\
\hline$\# 5$ & $4.1 \times 10^{-8}$ & $1.0 \times 10^{-10}$ & $5.0 \times 10^{-10}$ & $6.0 \times 10^{-10}$ \\
\hline
\end{tabular}

An interesting effect that can be attributed to TID effects concerns the received power level during the experiment. In this particular IR-UWB system, the received power can only be estimated by means of the measured channel impulse response power and the accumulation count of the frame preamble. Fig. 8 shows a possible degradation of the RF frontend over time with a drop of the received power of approx. $0.75 \mathrm{~dB}$. Due to the burn-in time, the DUTs have prior to the start of the experiment, temperature effects can be ruled out as a cause for this deviation. The on-chip temperature sensors of the DUTs show no significant change in temperature during irradiation.

Another parameter that confirms this degradation is the number of preamble sequence symbols needed by the receiver to receive the frame. In the IR-UWB PHY link, a preamble of pulses and a start frame delimiter (SFD) is sent to synchronize the receiver and indicate the start of a frame. A higher preamble sequence count of the receiver indicates that the detection of the SFD was harder to achieve. Fig. 9 shows the necessary preamble count needed to acquire the SFD sequence during the same experiment that Fig. 8 shows. This observation is in contrast with earlier tests of a narrow band agile RF transceiver, where this specific effect was not observed $[10 ; 11]$

No impact on the transceivers ranging performance was observed in the further examination of the experiment data.

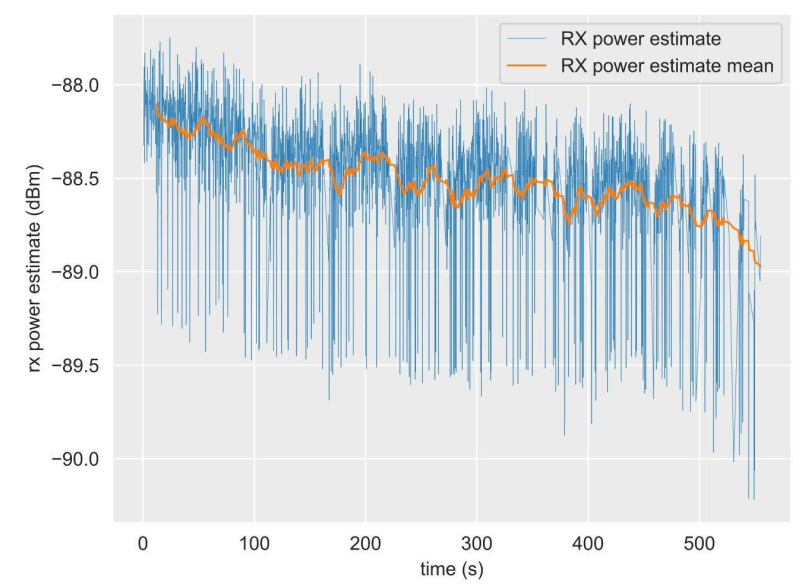

Fig. 8. Estimated received power of the initiator at $184 \mathrm{MeV}$ with a mean particle flux of $4.07 \times 10^{5}$ and a total fluence of $1.05 \times 9$. 


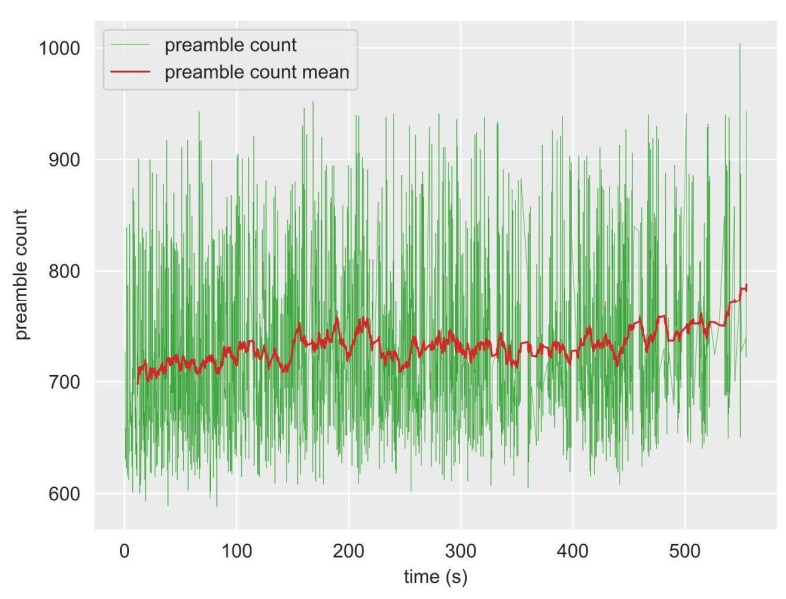

Fig. 9. Collected preamble sequences before SFD detection of the initiator at $184 \mathrm{MeV}$ with a mean particle flux of $4.07 \times 10^{5} \mathrm{\#} / \mathrm{cm}^{2} / \mathrm{s}$ and a total fluence of $1.05 \times 9 \# / \mathrm{cm}^{2}$.

Minor drops in the PDR between 92.3 - $98.6 \%$ were observed although these mostly correlate with the few register errors and mainly the lock-ups followed by hard resets of the DUTs.

\section{CONCLUSIONS}

In this paper, we presented the characterization of the DW1000 IR-UWB transceiver under radiation conditions, focusing on the degradative and damaging effects induced by TID and SEE. The results show a surprisingly strong performance considering that COTS hardware is used. The samples have shown some degradative effects. But they are acceptable to use the hardware in future LEO space missions for the deployment of wireless networks in spacecraft and launchers. In particular, no destructive effects were observed during the test campaigns. The data presented in this paper suggests a stable operation of this IC in a typical LEO mission is possible for tens of years. However, still, some more aspects need to be analyzed to fully characterize the performance and to reach a conclusion on the reliability of the hardware for critical space applications. In particular, these are:

1) For future SEE tests the controlling IC needs to be separated from the transceiver IC to have the majority of errors occur on the DUT instead of the microcontroller running the firmware for the test. On the board used during the SEE tests presented here both ICs are located in close proximity to each other which prevented a test setup where only the transceiver IC itself would be irradiated.
2) It is presumed that the receiver degradation seen during the SEE tests is attributable to TID effects occurring due to the prolonged proton irradiation. An additional TID test is planned to confirm this. Also, during a future SEE test, it could be examined, whether the observed effects are remedied by the annealing period.

\section{REFERENCES}

[1] W. H. Zheng and J. T. Armstrong, "Wireless intra-spacecraft communication: The benefits and the challenges," 2010 NASA/ESA Conference on Adaptive Hardware and Systems, Anaheim, CA, 2010, pp. 75-78, doi: 10.1109/AHS.2010.5546219.Iop

[2] M. Drobczyk, C. Strowik and C. Philpot, "A Wireless Communication and Positioning Experiment for the ISS Based on IR-UWB," 2017 IEEE Wireless Communications and Networking Conference (WCNC), San Francisco, CA, 2017, pp. 1-6, doi: 10.1109/WCNC.2017.7925487.

[3] M. Drobczyk and A. Lübken, "Novel wireless protocol architecture for intra-spacecraft wireless sensor networks (inspaWSN)," 2018 6th IEEE International Conference on Wireless for Space and Extreme Environments (WiSEE), Huntsville, AL, USA, 2018, pp. 89-94, doi: 10.1109/WiSEE.2018.8637342.

[4] A. N. Nikicio, W. Loke, H. Kamdar and C. Goh, "Radiation analysis and mitigation framework for LEO small satellites," 2017 IEEE International Conference on Communication, Networks and Satellite (Comnetsat), Semarang, 2017, pp. 59-66, doi: 10.1109/COMNETSAT.2017.8263574.

[5] Decawave., "DW1000 Datasheet," 2020 [Online]. Available: https://www.decawave.com/wpcontent/uploads/2020/04/DW1000_Datasheet.pdf

[6] IEEE Standard for Low-Rate Wireless Networks," in IEEE Std 802.15.4-2015 (Revision of IEEE Std 802.15.4-2011), vol., no., pp.1709, 22 April 2016, doi: 10.1109/IEEESTD.2016.7460875.

[7] ESA, "Single Event Effects Test Method and Guidelines, ESCC basic Specification No. 22900," Issue: 5, ESA, 2016

[8] H.Z.B., “Cobalt-60 source,” 2017 [Online]. Available: https://www.helmholtz-

berlin.de/angebote/ttindustrie/methoden/kobalt/index en.html [Accessed July 03, 2020]

[9] In-Circuit, "radino32 DW1000 Datasheet," 2020 [Online]. Available: https://wiki.in-circuit.de/images/6/63/305000092A_radino32_DW100 0.pdf [Accessed Jul 02, 2020]

[10] J. Budroweit and M. Sznajder, "Total Ionizing Dose Effects on a Highly Integrated RF Transceiver for Small Satellite Radio Applications in Low Earth Orbit," 2018 IEEE International Symposium on the Physical and Failure Analysis of Integrated Circuits (IPFA), $\quad$ Singapore, 2018, pp. doi: 10.1109/IPFA.2018.8452610.

[11] J. Budroweit and M. P. Jaksch, "In-Situ TID Testing and Characterization of a Highly Integrated RF Agile Transceiver for Multi-Band Radio Applications in a Radiation Environment," 2019 IEEE International Conference on Wireless for Space and Extreme Environments (WiSEE), Ottawa, ON, Canada, 2019, pp. 1-6, doi: 10.1109/WiSEE.2019.8920328. 